\title{
VEGA - Implementando um Laboratório Virtual Imersivo no OpenSim
}

\author{
Liane Tarouco $^{1}$, Bárbara Ávila ${ }^{1}$, Érico Amaral ${ }^{1,2}$, Herik Zednik ${ }^{1}$ \\ 1Programa de Pós-graduação em Informática na Educação - Universidade \\ Federal do Rio Grande do Sul (UFRGS) \\ ${ }^{2}$ Instituto Federal Sul-rio-grandense - Campus Santana do Livramento \\ lianeepenta.ufrgs.br, barbaragorzizaavila@gmail.com, \\ ericohoffamaralegmail.com, hzednikehotmail.com,
}

\begin{abstract}
Resumo. A pesquisa aqui relatada versa sobre a busca pela implementação de um laboratório virtual de Geometria dentro do metaverso OpenSim. Tendo em vista as limitações no que se refere aos objetos oferecidos pelo metaverso e à fluência necessária em programação para que seja possível o desenvolvimento de objetos para o OpenSim, foram exploradas ferramentas de autoria como recursos alternativos para se promover um desenvolvimento facilitado de objetos, que não requeira conhecimentos avançados em programação. Atualmente, tem-se explorado o Google SketchUp para a elaboração de objetos 3D a serem incorporados no metaverso com o apoio do viewer Firestorm. Em paralelo às pesquisas técnicas, foi desenvolvida uma estrutura pedagógica que define a forma como o laboratório deverá funcionar, com diferentes ambientes, objetos e atividades a serem realizadas.
\end{abstract}

Palavras-chave: Geometria. Realidade Virtual. Imersão. Interação

\begin{abstract}
The research reported here concerns the search for the implementation of a virtual laboratory of Geometry in the metaverse OpenSim. Given the limitations with regard to the objects offered by the metaverse and the necessary fluency in programming to make it possible to develop objects for OpenSim, authoring tools were explored as alternative resources to promote development facilitated the objects, which does not require advanced knowledge in programming. Currently, we have explored Google SketchUp for drawing 3D objects to be incorporated into the metaverse viewer with support from the Firestorm. Alongside the technical research, we developed a pedagogical structure that defines how the lab should work with different environments, objects and activities to be performed.
\end{abstract}

Keywords: Geometry. Virtual Reality. Immersion. Interaction

\section{Introdução}

Aproximar a geometria da realidade do aluno se caracteriza como um desafio apontado por muito educadores. Embora diferentes métodos e ferramentas estejam disponíveis aos professores com a finalidade de facilitar o ensino e a aprendizagem da geometria, diversos estudos apresentam um quadro desfavorável ao aprendizado desse conteúdo, bem como ao desenvolvimento de competências e habilidades. Isso se torna evidente ao 
analisar o resultado das avaliações oficiais divulgadas nos últimos anos, os quais remetem à necessidade de pesquisar e desenvolver materiais educacionais que incorporem as teorias pedagógicas nas estratégias de utilização.

Diante dessa realidade e compreendendo que a construção do conhecimento geométrico é um processo ativo, pesquisadores estão constantemente em busca de novos recursos e metodologias que contribuam com o processo de ensino $\mathrm{e}$ aprendizagem. Essa busca aponta para necessidade de inserir o aluno em atividades que incentivem a aprendizagem ativa, sejam atrativas e compreensíveis para ele, tais como jogos, simulações, brincadeiras, observações, leituras, exercícios, resolução de problemas, enfim, atividades desafiadoras e que provoquem o pensar, que permitam construir significados e conceitos, onde eles protagonizem de forma autônoma e interajam, produzindo um saber compartilhado e colaborativo.

Nesta experiência, buscou-se na realidade virtual uma forma de possibilitar ao usuário uma experiência bastante similar ao ambiente real, através da imersão em um mundo virtual. A simulação de situações vivenciadas no mundo real oferece ao aluno a oportunidade de experimentar diversos ambientes, cujas vantagens se destacam por questões de inacessibilidade, segurança ou economia. A combinação de um ambiente imersivo que apresente metas, objetivos e visualizações tridimensionais realistas, com uma simulação que enfatize a experimentação com base científica, oportuniza aos alunos a compreensão de conceitos científicos através da condução de experimentos cada vez mais próximos da realidade.

Potenciar a aprendizagem significativa através de situações nas quais o aluno aprenda a aprender, é a inquietação deste grupo de pesquisa (ZEDNIK, 2009). Dessa forma, tem se buscado desenvolver ferramentas mais atrativas, que possam apoiar professores e alunos no alcance dos objetivos educacionais e estimular o desenvolvimento de conceitos referentes à geometria analítica, plana e espacial. Assim, originou-se o projeto ora apresentado, com base num ambiente virtual de imersão, capaz de funcionar como um espaço de mediação para a aprendizagem. O objetivo é utilizar a realidade virtual para contextualizar a geometria de forma atrativa e dinâmica, onde os estudantes possam interagir com formas geométricas de forma ativa, estimulando a aprendizagem colaborativa através de uma postura autônoma diante desses recursos. "O ensino ativo presume que os alunos são pessoas capazes de dar significado às coisas, construtores ativos do seu próprio conhecimento; que trazem a cada experiência de aprendizagem uma reserva de informação da qual podem dispor ao procurarem compreender" (FREED, 1997).

Para implementar esse projeto, optou-se a partir da análise prévia de outros ambientes de imersão como Second Life e Wanderland, por utilizar o OpenSim (OS). Além de atender necessidades funcionais como: programação em várias linguagens, comunicação interativa, modelagem gráfica de objetos e importação de arquivos de imagem e áudio que permitiam alcançar variadas estratégias de imersão, o OS é um software de licença de uso livre, indo ao encontro das medidas adotadas pelo Governo Federal de incentivo à inclusão digital e à inovação através da adoção de modelos que privilegiem o uso do software livre, desde 2003.

\section{Laboratórios Virtuais e sua relação com a Teoria da Carga Cognitiva}

Muitos recursos computacionais vêm sendo utilizados no contexto do ensino de geometria. Softwares que permitem a construção e manipulação de objetos têm 
proporcionado o desenvolvimento da autonomia do alunado a partir de uma atuação ativa no processo de aprendizagem.

Neste artigo, é proposto um novo passo nestas práticas educacionais, adotandose o uso do metaverso OpenSim para a construção de laboratórios virtuais de geometria. A ideia de uso dos mundos virtuais como forma de explorar novas situações de ensino e aprendizagem ganhou força com o entendimento do grupo de pesquisa sobre a importância do uso de laboratórios de aprendizagem que permitam uma participação ativa do estudante nas atividades didáticas. Considerando-se que o acesso a laboratórios pode encontrar restrições em função de uma alta demanda de alunos para um escasso espaço físico que é comumente encontrado nas escolas, os Laboratórios Virtuais (LVs) passam a ser uma alternativa eficiente para o acesso a materiais pedagógicos interativos (AMARAL et al., 2011).

Através da manipulação de réplicas de objetos presentes no mundo concreto, o estudante pode se engajar em experimentos que simulam situações reais, fornecendo saídas baseadas nas ações realizadas pelos seus avatares. Embora Blascovich e Bailenson (2011) argumentem que a sensação de imersão e comprometimento com o mundo virtual seja muito mais acentuada quando se utiliza as tecnologias de tracking, acredita-se que a representação por avatares já seja um passo importante na direção do envolvimento pleno em um Laboratório Virtual. Esta convicção está fortemente amparada nas novas constatações feitas por Paas e Sweller na Teoria da Carga Cognitiva, relacionando habilidades de comunicação, imitação e uso de gestos com o desenvolvimento da cognição humana.

Pesquisas recentes de Paas e Sweller (2011) mostraram que atividades como a comunicação, o reconhecimento de movimentos humanos e o uso de gestos, decorrentes de motivações intrínsecas humanas, tornaram-se atividades primárias e já não requerem esforço cognitivo para o seu processamento na memória de trabalho. Além disso, constatou-se que as atividades primárias auxiliam no processamento das informações em atividades secundárias, que são decorrentes de uma aprendizagem estruturada, exploradas normalmente no contexto educacional e que necessitam de motivações externas para gerar um efetivo envolvimento dos estudantes.

Os autores apontam que o ser humano se utiliza da comunicação como um meio para apropriar-se e difundir conhecimentos, fato que já fora amplamente discutido na teoria sócio-histórica (VYGOTSKY, 2001; 2008). Paas e Sweller (2011) acrescentam que a comunicação funciona como base para o envolvimento em atividades colaborativas, com as quais os indivíduos unem as forças de sua memória de trabalho e tornam-se aptos a resolver problemas de maior complexidade.

No laboratório virtual em um ambiente imersivo, estudantes podem se utilizar de ferramentas como o chat para juntos discutirem e engajarem-se na resolução dos problemas propostos. Eles interagem entre si e com o mundo virtual através de seus avatares, o que lhes proporciona a sensação de compartilharem um ambiente, incentivando o seu envolvimento em atividades colaborativas.

Além da comunicação, uma atividade primária de suma importância para a promoção da aprendizagem é a imitação, sendo esta considerada a chave para a evolução humana na concepção de Tomasello (2003). Paas e Sweller (2011) afirmam que, ao assistir a realização de tarefas por outrem o indivíduo torna-se capaz de processar os passos realizados, reorganizando-os em esquemas a serem armazenados em sua memória de longo alcance. Salienta-se que é necessária uma base de conhecimento relacionada à tarefa em questão para que esta aptidão torne-se viável. 
Em mundos virtuais, o indivíduo pode observar a realização de tarefas efetuada por outros avatares e assim aprender com as mesmas. Além disso, pode-se ter acesso a demonstrações criadas a partir de animações disponibilizadas no ambiente.

A última atividade primária citada por Paas e Sweller (2011) diz respeito ao uso de gestos para melhor apropriação do conhecimento. Segundo os autores, a aprendizagem pode ser potencializada quando o ensino ocorre em uma mescla narrativa e gestual. Da mesma forma, o aprendiz tem maior capacidade de apropriação do conteúdo quando se utiliza de gestos ao longo de seu processo de aprendizagem.

Partindo-se desta premissa, atividades podem ser desenvolvidas em laboratórios virtuais, de modo que os estudantes sejam incentivados a gesticularem a partir de seus avatares enquanto interagem com os elementos contidos no ambiente. Ainda, se o laboratório dispuser de tecnologias adequadas para o tracking, mais eficaz e natural será a gesticulação, ampliando-se as possibilidades de desenvolvimento cognitivo do estudante, engajado em suas atividades de laboratório.

Embora o uso de metaversos como ambientes educacionais ainda seja uma iniciativa incipiente, muito devido a limitações técnicas, não somente no que diz respeito às máquinas e banda de internet, mas também pela falta de formação técnica docente para atuar com estas ferramentas, que ainda apresentam um certo grau de complexidade, ainda assim é promissora a sua atuação como ferramentas educacionais num futuro não muito distante. Tendo isso em vista, é importante que a academia voltese desde já para esta temática e busque desenvolver meios significativos de uso destes recursos no âmbito pedagógico.

\section{Ambientes imersivos: contextualização, possibilidades e limitações}

A proposta de aplicação de Laboratórios Virtuais na educação não é pioneira na Universidade Federal do Rio Grande do Sul (UFRGS). Uma iniciativa anterior, por exemplo, foi realizada no projeto ARCA (Ambiente de Realidade Virtual Cooperativo de Aprendizagem), oriundo de uma parceria com a Universidade Luterana do Brasil (ULBRA) e a Universidade Católica de Pelotas (UCPEL).

O projeto citado contou com o apoio do AWEDU (The Active Worlds Educational Universe), pertencente à empresa Active Worlds. O AWEDU proporcionava o acesso a uma comunidade acadêmica formada por instituições de diversos países. A participação das instituições era gratuita, bastando o encaminhamento de uma declaração de existência de projeto.

Com o ingresso da instituição no projeto, a Activeworlds disponibilizava um espaço no metaverso, avatares, objetos 3D e um kit para o desenvolvimento de software. $\mathrm{O}$ acesso ao mundo virtual era feito através de um navegador que também era fornecido sem custo às instituições (GUELLER et al., 2000). Entretanto, o projeto ARCA foi suspenso em virtude de que o AWEDU deixou de ser oferecido gratuitamente às instituições de ensino, o que veio a inviabilizar a continuidade da pesquisa. Este é um cenário que tem se buscado evitar com a adoção de uma ferramenta baseada em software livre, como o OpenSim.

Outra proposta de uso de mundos virtuais para estimular a aprendizagem encontra-se na tese de Medina (2004), sendo esta baseada na implementação de um Laboratório Virtual de Redes. O laboratório contava com um chatterbot chamado Electra, o qual havia sido programado para responder aos questionamentos dos alunos sobre conceitos relacionados a esta área de conhecimento. Os resultados da aplicação do 
laboratório e do apoio via chat permaneciam disponíveis 24 horas por dia aos estudantes e foram muito satisfatórios, conforme relata a autora.

Visando uma continuidade nos projetos relacionados ao uso da realidade virtual através dos metaversos, para propiciar o acesso a laboratórios de aprendizagem, buscase agora investir numa solução aberta em termos de mundo virtual. Um, dentre o conjunto de argumentos válidos para esta transição é o fato de que as comunidades de software livre vêm crescendo continuamente, o que propicia maior acesso a soluções de fácil implementação.

Allison et al. (2010) estabeleceram uma comparação entre um metaverso proprietário, o Second Life e o mundo virtual baseado em software livre, OpenSim , que fora utilizado no presente projeto. $\mathrm{Na}$ análise realizada, percebemos que a distância entre os servidores da Linden Labs (empresa responsável pelo SL), aumenta a necessidade de banda para a navegação, enquanto que no OS há a possibilidade de instalação de servidor na própria máquina do usuário, liberando espaço na rede. $\mathrm{O}$ acesso ao SL tem ainda uma restrição etária, que impõe a necessidade de seus usuários terem atingido, no mínimo, a idade de 18 anos para que possam se cadastrar no metaverso. Tal restrição dificulta, ou até inviabiliza, a aplicação destes recursos em atividades da Educação Básica. É importante lembrar que a proposição de um Laboratório Virtual de livre acesso requer uma ferramenta que possibilite a inclusão de estudantes de todas as idades (vantagem oferecida pelo OpenSim).

A Free Software Foundation (apud MIRANDA et al, 2005) destaca quatro tipos de vantagens para uso do software livre que ajudam a estabelecer uma relação de liberdade entre software e usuário, são elas: o usuário tem liberdade de executar o programa para qualquer finalidade; a liberdade de estudar e analisar como o programa funciona e adequá-lo às necessidades do usuário; a liberdade de disponibilizar novas versões, colaborando e ajudando outros usuários; a liberdade de modificar e aperfeiçoar o programa e disponibilizar as melhorias, beneficiando toda a comunidade. Razões pelas quais direcionou-se a implementação do Laboratório Virtual de Geometria para o OpenSim, metaverso baseado em software livre. Este direcionamento ocorreu não somente pelo acesso livre, independentemente dos objetivos de empresas, que podem sofrer alterações ao longo do tempo, seguindo as necessidades do mercado, mas também por abrir possibilidades que trazem a participação em comunidades de software livre, e que permite maior autonomia no uso da ferramenta e, tão logo, maior autonomia sobre o ambiente a ser construído.

\section{Linguagens de programação e Ferramentas de Modelagem para o OpenSim}

Leal (2007) aponta para o surgimento de vários mundos virtuais baseados em ambientes imersivos nestes últimos anos. Dentre os metaversos desenvolvidos encontra-se o OpenSim, que teve o início de sua implementação em meados de 2007.

Baseado em software livre, o OpenSim (OS) visava o oferecimento de um mundo 3D compatível com o já existente Second Life (SL), sendo este de licença proprietária. O principal objetivo do OpenSim foi disponibilizar um servidor 3D flexível e modular compatível com os viewers/clientes do Second Life. Além de permitir o suporte a múltiplos viewers, assim como o Second Life, o OpenSim ainda oferece a possibilidade de acesso simultâneo a um mesmo mundo virtual através de diferentes protocolos.

Visando-se o oferecimento de uma ferramenta espelhada no funcionamento do Second Life, o OpenSim foi idealizado de modo a carregar funcionalidades previamente 
desenvolvidas para o SL, porém expandindo as possibilidades de linguagens de programação com relação ao metaverso proprietário.

Enquanto que o Second Life conta apenas com sua linguagem própria (Linden Script Language - LSL), o OpenSim trabalha não somente com o LSL, mas também com a linguagem de scripts OpenSim Scripting Language (OSSL) (sendo esta uma extensão da Linden Script Language), e com as linguagens C\#, Java e Visual Basic. Salienta-se que os códigos podem ser executados de forma concorrente pelo servidor OpenSim, e são visualizados por todos os usuários do ambiente.

Morgado et al. (2011) esclarecem que a linguagem LSL apresenta uma sintaxe semelhante à linguagem $\mathrm{C}$, podendo ser aplicada no desenvolvimento de objetos em três dimensões, nos quais deverão ser aplicados códigos em LSL para disparar diferentes ações. Já a OSSL visa contemplar algumas funcionalidades que não encontram suporte na linguagem LSL. Como exemplos, apresentam-se as funções Teleport Agent, que permite levar um avatar de um ponto a outro no mundo virtual e a Set Dynamic Texture $U R L$ que possibilita ser exibida, no próprio metaverso, uma imagem externa gerada dinamicamente.

Além das várias ferramentas para o desenvolvimento de conteúdo, o OpenSim dispõe de funcionalidades que potencializam este metaverso como um espaço importante para a alocação de laboratórios virtuais. Dentre as funcionalidades do OS encontram-se os diferentes meios de comunicação disponíveis a partir do uso do áudio ou em conversas via chat. A construção de objetos gráficos e a disponibilização de recursos em multimídia também são funcionalidades que agregam valor ao ambiente quando este assume a forma de um LV.

O OpenSim permite a importação de objetos externos desenvolvidos a partir de ferramentas de autoria, como o Google SketchUp, que encontra-se em fase de avaliação nesta pesquisa. Além disso, discussões em fóruns destinados a trocas de informações sobre o metaverso apontam para a possibilidade de uso de outros aplicativos para a geração de conteúdo em 3D a serem incorporados ao OS, tais como o AutoCad, o Blender e o Flash. Os objetos devem ser renderizados e exportados para o formato .xml ou mesmo carregados para o metaverso a partir de suas extensões nativas. No caso do Google Sketch, pode-se exportar o conteúdo para o formato Collada (.dae) e importá-lo para o OpenSim através do viewer Firestorm, que dá suporte ao upload deste formato.

Encontrar os formatos adequados ao OpenSim e os viewers que lhes dão suporte ainda é uma tarefa de certa complexidade e que exige intensa pesquisa, pois o OpenSim ainda é um ambiente em fase de construção e, sendo este baseado em software livre, depende-se da iniciativa de programadores dispostos a investirem seus conhecimentos no desenvolvimento deste ambiente e compartilharem seus achados com os usuários deste metaverso.

A figura 01, logo abaixo, mostra o caminho para se exportar imagens produzidas no SketchUp para dentro do OpenSim através do viewer Firestorm. A seleção em vermelho destaca os tipos de objetos que podem ser importados para o ambiente.

Salienta-se que ainda se encontra certa dificuldade nesta importação, pois dependendo do grau de complexidade da imagem gerada no SketchUp, tem-se uma desconfiguração quando a mesma é importada para o OS. Além disso, ainda há problemas com relação ao reconhecimento da imagem como um objeto deste metaverso. 


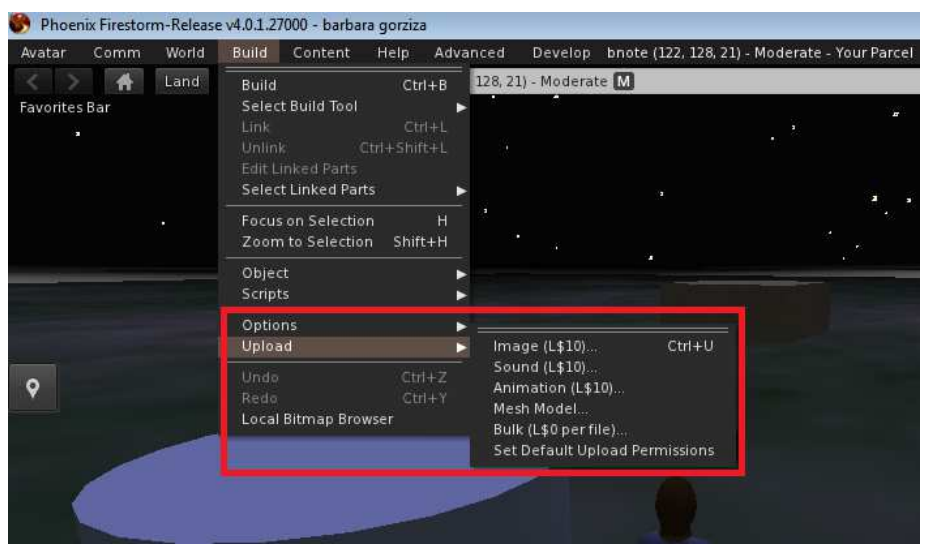

Figura 01 - Viewer Firestorm com opções de importação de objetos selecionada

Várias aplicações construídas a partir de diferentes ferramentas de autoria poderão ser utilizadas nos mundos virtuais. Em paralelo à pesquisa com o SketchUp, estão em fase de testes objetos desenvolvidos em flash, com arquivos .swf sendo carregados para o ambiente através de páginas web, aplicadas como texturas a objetos do próprio OpenSim. Outro estudo em fase de teste é a aplicação de objetos desenvolvidos no Geogebra como texturas em objetos do OS. O Geogebra possibilita a elaboração de objetos que exploram o conhecimento algébrico e geométrico, e que promovem, a partir da experimentação, a formulação de conjecturas e testagem de propriedades matemáticas.

Conforme já mencionado, o processo de integração destes diferentes objetos está diretamente relacionado aos viewers utilizados no OS. Esta conclusão se deu a partir de inúmeros testes com diferentes visualizadores como: o Hippo, o Imprudence viewer, o Firestorm e o RealXtend. Todas estas ferramentas são de código aberto, disponíveis para o acesso aos mundos virtuais. Durante os testes, o Hippo e o Imprudence Viewer apresentaram restrições na importação e configuração de objetos. Em contrapartida, nos testes envolvendo o RealXtend e Firestorm viewer, o processo de importação de objetos pode ser realizado de forma simplificada, porém, com os percalços já descritos nesta seção.

\section{Estratégias do Projeto}

Para a idealização do Laboratório Virtual VEGA (Virtual Environment for Geometry Acquaintance) foi organizado um conjunto de atividades envolvendo a Geometria, compreendendo de uma abordagem mais básica até o trabalho com a resolução de problemas mal estruturados, explorados em pesquisas como de Wijekumar e Jonassen (2004).

O foco principal das atividades a serem implementadas neste laboratório será o estímulo das noções espaciais do estudante através da sua participação nos desafios propostos. Neste contexto, tem se levado em consideração os níveis de interatividade do LV que deverão influenciar diretamente no processo de aprendizagem de seus usuários (AMARAL et al., 2011). Tendo em vista que, quanto maior a interatividade da ferramenta, maior o envolvimento de seus usuários, busca-se nesta proposta atingir o maior nível de interatividade possível. E este poderá ser alcançado com o uso de ambientes imersivos (os metaversos), representados aqui pela figura do OpenSim. 
Para organizar as atividades laboratoriais, foi estruturado um contexto didático, apoiado no ensino da geometria, dividido em três diferentes momentos.

A primeira etapa foi desenhada com o intuito de compreender conceitos básicos relacionados à geometria. Nesta fase devem ser desenvolvidos os temas de base sobre área, volume, coordenadas e fórmulas aplicadas.

Num segundo momento, objetiva-se a consolidação dos conhecimentos vivenciados na etapa anterior. Para isso, deverá ser implementado e disponibilizado pelo professor um repositório de formas geométricas, o qual ficará à disposição dos alunos. Este repositório, por sua vez, deverá ser desenvolvido com o apoio de ferramentas de autoria, como aquelas citadas na seção anterior deste artigo. Ainda nesta etapa, os estudantes deverão manipular os objetos e responder aos desafios lançados no ambiente. Além disso, serão realizadas medições e a construção de novos objetos geométricos com as formas já existentes.

$\mathrm{Na}$ terceira etapa deverá constar a aplicação prática dos conhecimentos construídos nas etapas anteriores. O objetivo desta fase será de aguçar o raciocínio e a visão espacial dos alunos, com base nos conceitos estudados nos momentos passados. A resolução de problemas será uma tarefa-chave nesta fase do estudo.

\subsection{Implementação e Discussões}

A estrutura do modelo desenvolvido sobre o OpenSim disponibiliza um Mundo Virtual e imersivo para o ensino da geometria. A Figura 02 apresenta um roteiro criado para a demonstração da estrutura pedagógica adotada neste projeto.

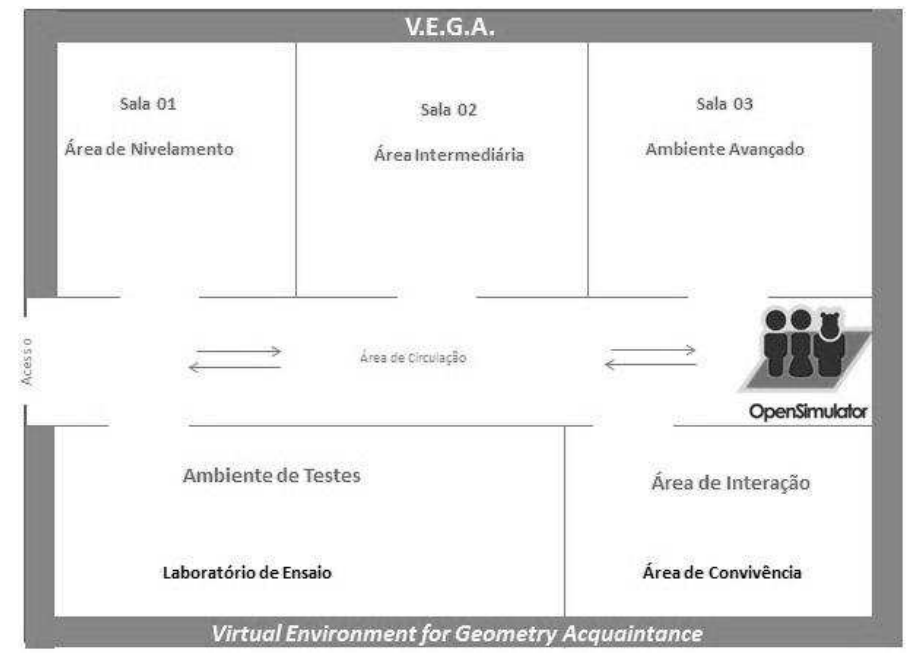

Figura 02 - Proposta de espaço de imersivo

Abaixo seguem aa descrição de cada sala que compõe a estrutura do ambiente, sugestionando um roteiro natural a ser seguido pelo aluno durante o processo de construção do conhecimento:

- Os assuntos que abordam os primeiros conceitos de geometria são apresentados na Área de Nivelamento (Sala 01). Esta sala é um espaço destinado a equalização do conhecimento, acerca do tema, onde objetos interativos são disponibilizados, como links para sites web, tutoriais sobre geometria e vídeos educacionais.

- No segundo ambiente (Sala 02) o estudante terá acesso a um repositório de figuras geométricas e tutoriais sobre a carga/importação das figuras para o ambiente. $\mathrm{O}$ 
intuito é fornecer meios para que o aluno experimente seus conhecimentos prévios sobre geometria a partir de atividades elaboradas com foco na interação aluno/objeto de estudo.

- A Sala 03 é destinada a ser um espaço para a construção, estabelecendo uma relação prática entre os conceitos estudados e os objetos do mundo real. Será atribuída a cada aluno uma área, no laboratório de ensaio, com o intuito de fornecer um espaço para seus experimentos.

- O Laboratório de ensaio, uma área aberta, onde o estudante deverá possuir controle total do ambiente, com autorização para edição, construção e importação de novos objetos.

- O Espaço de Convivência é caracterizado por um ambiente aberto no qual o aluno poderá interagir com os demais colegas. Serão oferecidos recursos como: mural de ideias arquitetônicas para a composição dos ambientes, acesso a Internet, espaço de interação com figuras geométricas e links para repositórios de objetos disponíveis para OpenSim.

- Vislumbrando que este projeto se torne atraente ao aluno, além dos objetos pedagógicos dentro de casa sala serão disponibilizados áreas e itens de conforto. Além disso, será permitida a carga de objetos educacionais criados em diferentes ferramentas de autoria como: Autocad, Blender, Sketch-up, Geogebra e html, fornecendo aos alunos recursos complementares, sempre voltados para as atividades desenvolvidas naquele espaço.

- Também existirá uma área de circulação para o acesso aos ambientes. Neste ponto, é importante salientar que os estudantes poderão se deslocar de forma autônoma pelos cômodos, de acordo com sua necessidade e nível de compreensão, podendo ir e vir sempre que acharem conveniente, ou seja, não existirá uma hierarquia ou sequência linear para a realização das atividades.

A construção do projeto, em relação ao roteiro disponibilizado ao aluno, está de acordo com o método de aprendizagem através da experimentação proposta por Kolb (2000): na Sala 01, com a contextualização do tema, temos a experiência concreta; a observação reflexiva, na sala 02 , com a possibilidade de construção de novos objetos a partir de figuras geométricas; sobre a conceituação, a Sala 03 e o Laboratório de Ensaio, focam na atividade prática a fim de estimular os estudantes; por fím a experimentação ativa também é percebida na Sala 03 e Laboratório de Ensaio, visto que nestes ambientes serão atingidos os estágios da experimentação ativa.

\section{Considerações finais}

A proposta de um Laboratório Virtual de Geometria, trazida neste artigo, vislumbra despertar o interesse dos alunos para a aprendizagem da matéria em questão, aproximando-a de suas aplicações no mundo real. Isso se efetiva no momento em que os estudantes se utilizam de uma ferramenta capaz de construir réplicas, trazendo elementos cada vez mais realísticos, semelhantes aos inúmeros jogos com os quais eles já estão habituados.

Salienta-se que, no contexto do projeto, o estudo e construção foram fundamentados sobre os princípios das boas práticas de educação digital, culminando em uma proposta de roteiro pedagógico sobre um mundo virtual, que de acordo com Kolb (2000) atende as etapas necessárias em um ciclo de aprendizagem.

A pesquisa aponta que a utilização de ferramentas de imersão incorpora muitos elementos associados à teoria construtivista e fornece uma estrutura adequada ao 
aprendizado, incluindo aprendizagem ativa (construção de personagens e modificação do mundo virtual), interação social (comunicação e interação com outros alunos) e solução de problemas (observação de processos e aplicação de conhecimentos do mundo real a simulações no mundo artificial). Assim, os recursos tecnológicos digitais não só redimensionam as condições de acesso às fontes de informações, como também ampliam as possibilidades de aprendizagem, através do uso de simulações, manipulações simbólicas e múltiplas formas de representação (WILEY, 2001).

\section{Referências}

ALLISON, Colin; MILLER, Allan; STURGEON, Thomas; NICOLL, J. Ross. Educationally Enhanced Virtual Worlds. 40 th ASEE/IEEE Frontiers in Education Conference. p. 1-5, 2010.

AMARAL, Erico; AVILA, Barbara; ZEDNIK, Herik; TAROUCO, Liane. Laboratório Virtual de Aprendizagem: Uma Proposta Taxonômica. Renote. v. 10 n. 2, p. 1-10, 2011.

BLASCOVICH, Jim; BAILENSON, Jeremy. Avatars, eternal life, new worlds and the dawn of the virtual revolution: Infinite Reality. New York: HarperCollins, 2011.

GUELLER, Marlise; PASSERINO, Liliana M.; TAROUCO, Liane M. R.; SILVEIRA, Sidnei. Aprendizagem e avaliação em um Ambiente de Realidade Virtual Cooperativo de Aprendizagem (Projeto ARCA). In: Rede Iberoamericana de Informática Educativa. Viña del Mar, 2000, p. 1-22.

KOLB, David A.; BOYATZIS, Richard E.; MAINEMELIS, Charalampos. Experiential Learning Theory: Previous Research and New Directions. In: LEAL, David (2007). Mundos Virtuais On-line: um mini-guia. Disponível em http://www.masternewmedia.org/pt/2007/04/10/mundos_virtuais_online_um_miniguia. htm. Acessado 12/12/2011.

LEAL, David (2007). Mundos Virtuais On-line: Um Mini-guia. Disponível em http://www.masternewmedia.org/pt/2007/04/10/mundos_virtuais_online_um_miniguia. htm. Acessado 12/12/2011.

MEDINA, Roseclea D. ASTERIX - Aprendizagem significativa e tecnologias aplicadas no ensino de redes de computadores: integrando e explorando possibilidades. Porto Alegre: PGIE da UFRGS, 2000. 174 p. Tese de doutorado Programa de Pós-graduação em Informática na Educação, Universidade Federal do Rio Grande do Sul, 2000.

MIRANDA, Isabella Tamine Parra et al. Softwares Livres: vantagens. Maringa Management:Revista de Ciências Empresariais, v. 2, n.1, p. 26-33, jan./jun. 2005.

MORGADO, Leonel Caseiro; BARBOSA, Luis; SOUZA, Antonio. Ensaio de utilização do mundo virtual Second Life no ensino de programação de computadores. UTAD - Universidade de Trás-os-Montes e Alto Douro, Portugal, 2011. 
PAAS, Fred; SWELLER, John. An Evolutionary Upgrade of Cognitive Load Theory: Using the Human Motor System and Collaborationt: o Support the Learning of Complex Cognitive Tasks. In: Educ Psychol Rev. New Jersey, 2011, p. 1-19.

VYGOTSKY, Lev S. A construção do pensamento e da linguagem. São Paulo: Martins Fontes, 2001.

WIJEKUMAR, Kausalai K; Jonassen, David H. The role of computer tools in experts' solving ill-structured problems. Computers in Human Behavior. v. 23, p. 664-704, 2004.

ZEDNIK, H. Análise do uso de um de objeto de aprendizagem de física na compreensão de conceitos matemáticos. In: Francisco Kennedy Silva dos Santos. (Org.). II Colóquio abrindo trilhas para os saberes: formação humana, cultura, diversidade. 01 ed. Fortaleza: SEDUC, 2009, v. 01, p. 208-219. 